\title{
Smart air purifier suitable for small public spaces
}

\author{
Pawel Idziak ${ }^{1, *}$, Michal Gojtowski ${ }^{2}$ \\ ${ }^{1}$ PUT Poznan University of Technology, Department Electrical Engineering Poznan ul. Piotrowo 3A Poland \\ ${ }^{2}$ WUT Production Department, 91944 Les Ulis Cedex A, France
}

\begin{abstract}
The article presents the concept and implemented, functional physical model of a mobile air purifier for small public spaces or apartments. The purifier is a compact, controlled by microcontroller of the Arduino UNO series. The model is equipped with a set of sensors allowing to determine the content of PM 2.5 contaminants. After exceeding the adopted threshold in the software, the system automatically starts the process of air filtering. The following factors are measured: solid contamination content, humidity and air temperature as well as pressure drop on built-in filters. The purifier absorbs solid pollutants and reduces VOC pollutants. The system has been equipped with a screen informing the user about air parameters and the state of the filter contamination.
\end{abstract}

\section{Introduction}

An avalanche-like pollution of the natural environment, including air, has a negative effect on living organisms. This is especially true for residents of large urban agglomerations. The exposures are subjected to residents staying both in the open area and in enclosed spaces. Commonly used room ventilation systems in most cases do not have an appropriate mechanism for monitoring and cleaning the air entering the building $[1,2,3]$. The idea motivating to engage in this problem is the contrivance of providing users, of said above building objects, the access to air devoid of mechanical and chemical pollution, negatively affecting human health (in accordance with the recommendations of the WHO.

\section{The choice of air filtration method}

Filter systems should ensure mechanical separation and demonstrate the ability to capture or neutralize unwanted chemical compounds. The methods of air purification (filters) can be classified due to the method of elimination of unwanted inclusions on filters: mechanical, chemical, ionizing, thermodynamic, photocatalytic and hydrous.

In air cleaning systems, the most popular are mechanical filters, specially include HEPA type filters (High Efficiency Particulate Air Filter). The properties of these filters are defined in PN EN 1822: 2009 $[3,4]$.The main method of removing volatile organic compounds VOCs from the air is the use of a carbon filter. VOCs are captured from the air by bonding impurities through the activated carbon. The principles of operation and the exploitation of these filters are described in the literature $[1,2]$.

\section{Concept of mobile air purifier}

The project assumes the development of a system that supplies air to the room from the outside with unknown pollination. The process of air quality improvement will be based on reactive air purification by the device in case of detecting an abnormal concentration of one of the detected contaminants.

The design assumes that the filter will be mobile, with the capacity to improve the state of air in rooms with a volume of about $130 \mathrm{~m}^{3}$ (approx. $50 \mathrm{~m}^{2}$ ). It was assumed that: the sensory system should monitor air dustiness, temperature, humidity and pressure drop across the device's filters; the device switch on automatically, information about air condition, temperature and humidity should be provided to the user via LCD display, the filtering system should trap particles PM 2.5 with the efficiency of $\min .95 \%$ and the used fan should provide air flow of $\mathrm{min} .100 \mathrm{~m} 3 / \mathrm{h}$, due to economic aspects, it was assumed that the accuracy of the used sensor will only allow approximate measurements, therefore it will be necessary to use an additional button activating the filtering system regardless of the sensor's indications, the filtering system should be monitored, i.e. the user should be informed about the necessity of replacing of the filter cartridges, e.g. with an appropriate message on the display, unit cost of the device should not exceed $250 €$.

Originally, it was assumed that the system's operation algorithm should take into account the signals from measurement transducers detecting the concentration of PM 2.5 dusts, formaldehyde, oxide and carbon dioxide. Lack of coupling with the ventilation system means that the system will not be able to reduce $\mathrm{CO}$ and $\mathrm{CO} 2$ concentrations. For economic reasons, $\mathrm{CO}, \mathrm{CO} 2$ and formaldehyde sensors have been abandoned. It was assumed that the cleaning system should be equipped 
with two basic filters, i.e. a HEPA filter and a filter saturated with active carbon.

\section{Construction of the purifier}

To reduce the cost of the prototype, an Arduino UNO development board equipped with an Atmega328F-PU microprocessor was used. The program uses subprograms dht11.h, dustSensor.h, pressureSensor.h and LiquidCrystal.h intended for handling dust sensors, temperature sensors and LCD display $[5,6]$. In the main loop of the program three functions are called successively, each of which corresponds to one of the used sensors. Then the program is stopped by the delay function for a period of 2 seconds after which the next program loop is executed.

The void dht11 () function supports the temperature and humidity sensor. Inside this block an operation is performed to collect data from the sensor, display them on the screen of the device and (optionally) send the signal in digital form to the computer. The void dustSensor () function provides support for an analog optical dust sensor. Its activation requires first the ignition of the IR diode for $0.28 \mathrm{msec}$. The measurement is performed a bit later. A condition has been introduced to the program, the fulfillment of which results in a change of the message on the LCD and switching on the fan. Another function is the void pressureSensor() function. It allows reading the analog value from the pressure sensor and assigning the value read from the transducer to the total variable. Then the condition is checked, the exceeding of which results in displaying information on the LCD display.

The cleaning system is activated when contaminants are detected. The dust transducer uses the phenomenon of scattering of light emitted by an infrared diode. The project uses a measurement module with a GP2Y1010AU0F sensor. The output measurement signal is a voltage analog signal $[5,6,7]$. Because the transmitter output signal didn't remain constant it was necessary to use additional supporting capacitor voltage.

The DHT11 module was used to measure temperature and humidity. This module is the double resistive sensor. The operating parameters of this module are: $0-50^{\circ} \mathrm{C}$, and (20-90)\% RH (Relative Humidity) [7]. The system is equipped with a microcontroller that collects signals from sensors and supports serial data transmission in the form of a digital "one-wire" type.

The purifier is equipped with two main filters, i.e. a mechanical EPA filter and a carbon filter. Both of these elements are clogged by the contaminants they pick up. This limits the air flow and thus increases the pressure before the filter system. Overpressure that appears before the filter is measured and compared to atmospheric pressure. The measurement of the pressure drop on the filter was carried out with the XCA505GN piezoresitive sensor [7].

As a fan drive, the BLCD motor was used. It drives the fan with a diameter of $250 \mathrm{~mm}$. Motor power is 3.2 $\mathrm{W}$, and the flow rate $232 \mathrm{~m}^{3} / \mathrm{h}$ at a speed of $800 \mathrm{rpm}$. The change of flow can be realized by changing the voltage.
It was assumed that the structure should ensure the stability of the entire device, so that accidental overturning of the filter is impossible. The filter cartridges of the cleaner are elements subject to wear. They are planned for easy disassembly and replacement. Figure 1 shows a sketch of the constructed device and a photo of the model. The first element of the cleaning system is a prefilter that catches the larger size of pollutants. Above is a fan forcing air movement in the system. The HEPA filter kit and the carbon filter were installed in succession. The last element is the net protecting the system from accidental damage. The casing is made of PVC pipe.

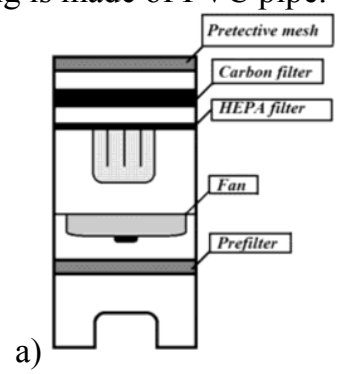

b)

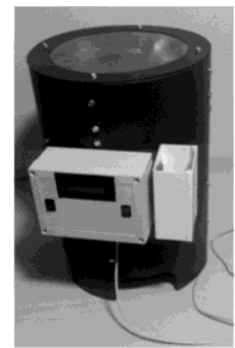

Fig. 1. Diagram of arrangement of elements inside the filter (a) and photo of the model (b).

\section{Observations and conclusions}

The cost of a single device operating in the concept of a distributed cleaning system is estimated at $240 €$. The operating costs of the system with independent cleaning devices are limited to replacing the filter cartridges and to the energy costs consumed by the devices. It is anticipated that filters should be replaced every 12-24 months. The estimated capacity of the devices is about $50 \mathrm{~W}$, which assuming an energy price of $0.23 € / \mathrm{kWh}$ and an 8-hour working day is approximately $25 €$ year.

The built-in device allows continuous monitoring of air dustiness and the start of the cleaning system after exceeding the allowed limit. For the constructed device this level was defined as $80 \mu \mathrm{g} / \mathrm{m}^{3}$.

Noise emission tests carried out in accordance with the requirements of many normative acts, e.g. PN-EN 60704-1: 2010 standard show that the sound pressure level emitted by the air cleaner does not exceed $30 \mathrm{~dB}$ (A), which is a lower value then the emission of commercial filters.

\section{References}

1. Ingo Nietzold, Air filtration (polish edition), Wydawnictwo Arkady, Warsaw 1984r.

2. https://aero7.pl (05.10.2018)

3. Standard PN EN 1822:2009 Air filters

4. Regulation of the European Parliament WE 1272/2008 (CLP)

5. https://www.dfrobot.com (13.12.2018)

6. https://www.systechillinois.com (16.12.2018r)

7. Catalog note of the sensors:, GP2Y1010AU0F, XCA505GN, DHT11 